\title{
Reply to Comment on 'Effect of ionization on ion acoustic solitary waves in a collisional dusty plasma' by J. Vranjes et al.
}

\author{
SAMIRAN GHOSH \\ Government College of Engineering and Textile Technology, 4 Cantonment Road, \\ Berhampore, Murshidabad 742101, West Bengal, India \\ (sran_g@yahoo.com)
}

(Received 5 December 2005)

The main objective of the Comment made by J. Vranjes et al. is that the sourcesink approach in studying physical phenomena, such as the ionization instability, is suspect. Also the term $\left(Q_{\mathrm{i}}-\nu_{\mathrm{L}} n_{\mathrm{i}}\right) v_{\mathrm{i}}$ in the ion momentum conservation equation of the source-sink model used by [1] is improper.

In reply, first of all we want to say that the source-sink model used by [1] is an adopted model. In fact, Johnson et al. formulated a one-dimensional sourcesink model for ionization instability in order to account for the low-frequency ionacoustic wave observed in their experiment [2]. After that D'Angelo [3,4] extended this model to account for low-frequency (ion-acoustic wave and dust-acoustic wave) ionization instabilities in a dusty plasma on the basis of the experimental conditions [2]. Later Wang et al. extended this model with more theoretical refinements on the basis of their experimental observations [5]. The model developed by Wang et al. [5] explains well the physical phenomena such as 'formation of voids' [6], 'ionization instability' $[3,4,7]$ etc. in the linear regime of a dusty plasma. We adopt this well-established model [5] to study the effect of ionization on nonlinear ionacoustic waves in a collisional dusty plasma. Hence, we do not like to make any comment regarding the justification of the model and also the genesis of the term $\left(Q_{\mathrm{i}}-\nu_{\mathrm{L}} n_{\mathrm{i}}\right) v_{\mathrm{i}}$ in the ion momentum conservation equation.

Also, the presence of the term $\left(Q_{\mathrm{i}}-\nu_{\mathrm{L}} n_{\mathrm{i}}\right) v_{\mathrm{i}}$ in the ion momentum conservation equation in [5] does not have any qualitative effects on 'formation of voids' or 'ionization instability', but has very small quantitative effects on the results in the linear theory (J. Goree, personal communication, December 2005). Thus, exclusion of this term from the ion momentum conservation equation does not change the physics of formation of voids or ionization instability (J. Goree, personal communication, December 2005).

Furthermore, one can easily find from our study [1] that the term $\left(Q_{\mathrm{i}}-\nu_{\mathrm{L}} n_{\mathrm{i}}\right) v_{\mathrm{i}}$ present in the ion momentum conservation equation has no effect on the result: owing to the scaling of $\nu_{\mathrm{L}}\left(O\left(\epsilon^{3 / 2}\right)\right)$ and by virtue of the relation between $Q_{\mathrm{i}}$ and $\nu_{\mathrm{L}}$ (equation (11) of [1]), the term $\left(Q_{\mathrm{i}}-\nu_{\mathrm{L}} n_{\mathrm{i}}\right) v_{\mathrm{i}}$ contributes only in higher order of $\epsilon\left(O\left(\epsilon^{5 / 2}\right)\right.$ and higher $)$, but the term $O\left(\epsilon^{5 / 2}\right)$ arising from $\left(Q_{\mathrm{i}}-\nu_{\mathrm{L}} n_{\mathrm{i}}\right) v_{\mathrm{i}}$ cancels out 
because of $Q_{\mathrm{i} 0}^{\prime}=\nu_{\mathrm{L}} n_{\mathrm{i} 0} \Rightarrow Q_{\mathrm{i} 0}=\nu_{\mathrm{L}}$ (equation (8) of [1]). Thus, if one drops this term from ion momentum conservation and studies the effect of ionization instability on small amplitude ion-acoustic solitary waves by the reductive perturbation technique, our result will be intact. Hence, the term $\left(Q_{\mathrm{i}}-\nu_{\mathrm{L}} n_{\mathrm{i}}\right) v_{\mathrm{i}}$ has no effect on ion-acoustic solitary waves in the presence of ionization at least in the small amplitude limit.

However, according to the experimental conditions (J. Goree, personal communication, December 2005; N. D’Angelo, personal communication, December 2005), we make the following assumptions.

(i) The neutrals are immobile, i.e. the dynamics of the neutrals are neglected as the mass per unit volume of the neutrals is much larger than that of the plasma. Also the degree of ionization is very low so that the variation of neutral density itself does not contribute significantly to the wave motion.

(ii) The effect of charge exchange is also neglected as it is insignificant according to experimental conditions.

(iii) The new ions are created through ionization of neutral gas by fast electrons. The electron collisions do not provide a Maxwellian of the tail so that the ion creation term $Q_{\mathrm{i}}=\sigma n_{\mathrm{n}} \psi$, where $\sigma$ is the ionization cross-section and $\psi$ the flux of ionizing electrons whose density is much smaller than the density of the thermal electrons $\left(n_{\mathrm{e}}\right)$. Actually, $Q_{\mathrm{i}}$ is the number of created ions per unit volume per unit time, so that $\nu_{\mathrm{ion}}=Q_{\mathrm{i}} / n_{\mathrm{i}}$ is the ionization frequency.

(iv) The ion losses are caused by the diffusion to the walls of the plasma container and absorption by the dust grains within the plasma and $\nu_{\mathrm{L}}$ is the ion loss frequency.

Thus, in this system the rate of change of ion number densities owing to ionizationloss mechanisms is given by

$$
\left(\frac{\delta n_{\mathrm{i}}}{\delta t}\right)_{\text {ionization-loss }}=\left(\frac{\delta n_{\mathrm{i}}}{\delta t}\right)_{\text {ionization }}-\left(\frac{\delta n_{\mathrm{i}}}{\delta t}\right)_{\text {loss }}=\nu_{\mathrm{ion}} n_{\mathrm{i}}-\nu_{\mathrm{L}} n_{\mathrm{i}} .
$$

Also the rate of momentum loss of ion fluid owing to various kinds of elastic and inelastic collisions is given by

$$
\left(\frac{\delta\left(m_{\mathrm{i}} n_{\mathrm{i}} v_{\mathrm{i}}\right)}{\delta t}\right)_{\mathrm{el} / \text { inel }}=m_{\mathrm{i}} v_{\mathrm{i}}\left(\frac{\delta n_{\mathrm{i}}}{\delta t}\right)_{\mathrm{el} / \text { inel }}+m_{\mathrm{i}} n_{\mathrm{i}}\left(\frac{\delta v_{\mathrm{i}}}{\delta t}\right)_{\mathrm{el} / \text { inel }} .
$$

In this case, for elastic collision

$$
\left(\frac{\delta n_{\mathrm{i}}}{\delta t}\right)_{\mathrm{el}}=0, \quad\left(\frac{\delta v_{\mathrm{i}}}{\delta t}\right)_{\mathrm{el}}=\nu_{\mathrm{i}} v_{\mathrm{i}}
$$

where $\nu_{\mathrm{i}}\left(=\nu_{\text {in }}+\nu_{\text {id }}\right)$ is the collision frequency of ions with neutrals and dust grains, whereas for inelastic collision

$$
\begin{gathered}
\left(\frac{\delta n_{\mathrm{i}}}{\delta t}\right)_{\text {inel }}=\left(\frac{\delta n_{\mathrm{i}}}{\delta t}\right)_{\text {ionization-loss }}=\nu_{\mathrm{ion}} n_{\mathrm{i}}-\nu_{\mathrm{L}} n_{\mathrm{i}} \\
\left(\frac{\delta v_{\mathrm{i}}}{\delta t}\right)_{\text {inel }}=\left(\frac{\delta v_{\mathrm{i}}}{\delta t}\right)_{\text {ionization }}=\nu_{\mathrm{ion}} v_{\mathrm{i}}
\end{gathered}
$$


This happens because of the fact that there is momentum loss of the ions that are created with zero momentum and the ions that are lost are no longer part of the ion family.

Next we consider the following Boltzmann-Vlasov equation for ions

$$
\frac{\partial f_{\mathrm{i}}}{\partial t}+\mathbf{v} \cdot \frac{\partial f_{\mathrm{i}}}{\partial \mathbf{x}}+\frac{e \mathbf{E}}{m_{\mathrm{i}}} \cdot \frac{\partial f_{\mathrm{i}}}{\partial \mathbf{v}}=\left(\frac{\delta f_{\mathrm{i}}}{\delta t}\right)_{\mathrm{el}}+\left(\frac{\delta f_{\mathrm{i}}}{\delta t}\right)_{\mathrm{inel}}
$$

Taking the zeroth $\mathbf{v}$ moment of (5), one gets (like J. Vranjes et al.) the following one-dimensional ion continuity equation

$$
\partial_{t} n_{\mathrm{i}}+\partial_{x}\left(n_{\mathrm{i}} v_{\mathrm{i}}\right)=\nu_{\mathrm{ion}} n_{\mathrm{i}}-\nu_{\mathrm{L}} n_{\mathrm{i}}=Q_{\mathrm{i}}-\nu_{\mathrm{L}} n_{\mathrm{i}} .
$$

Similarly taking the first $\mathbf{v}$ moment of (5) and using the equation of continuity to bring the equation of motion for ions to its standard form, one gets the following one-dimensional equation

$$
\begin{aligned}
n_{\mathrm{i}}\left(\partial_{t} v_{\mathrm{i}}+v_{\mathrm{i}} \partial_{x} v_{\mathrm{i}}\right) & =-n_{\mathrm{i}} \partial_{x} \phi-\sigma_{\mathrm{i}} \partial_{x} n_{\mathrm{i}}-\nu_{\mathrm{i}} n_{\mathrm{i}} v_{\mathrm{i}}-\nu_{\mathrm{ion}} n_{\mathrm{i}} v_{\mathrm{i}} \\
& =-n_{\mathrm{i}} \partial_{x} \phi-\sigma_{\mathrm{i}} \partial_{x} n_{\mathrm{i}}-\nu_{\mathrm{i}} n_{\mathrm{i}} v_{\mathrm{i}}-Q_{\mathrm{i}} v_{\mathrm{i}}
\end{aligned}
$$

This shows that there should not be the term $\nu_{\mathrm{L}} n_{\mathrm{i}} v_{\mathrm{i}}$, i.e. there should be the term $Q_{\mathrm{i}} v_{\mathrm{i}}$ instead of $\left(Q_{\mathrm{i}}-\nu_{\mathrm{L}} n_{\mathrm{i}}\right) v_{\mathrm{i}}$ in the ion momentum conservation equation (4) of [5] as well as in [1].

Thus, in the nonlinear regime, owing to the presence of the only term $Q_{\mathrm{i}} v_{\mathrm{i}}$ instead of $\left(Q_{\mathrm{i}}-\nu_{\mathrm{L}} n_{\mathrm{i}}\right) v_{\mathrm{i}}$ in the ion momentum conservation equation (3) of [1], there will be the extra term $\nu_{\mathrm{L}} V_{\mathrm{i}}^{(1)}$ (in accordance with the notation of [1]) in the left-hand side of equation (17) of [1]. As a consequence the expression of $\gamma$ will be

$$
\gamma=\frac{\nu_{\mathrm{i}}}{2}-\frac{\nu_{\mathrm{L}}}{2}\left(\delta \frac{\Delta \sigma}{\sigma_{0}}-2\right)
$$

instead of $(23)$ of $[1]$ and hence the condition for growth $(\gamma<0)$ will be

$$
\nu_{\mathrm{i}}<\nu_{\mathrm{L}}\left(\delta \frac{\Delta \sigma}{\sigma_{0}}-2\right)
$$

whereas the condition for damping $(\gamma>0)$ will be

$$
\nu_{\mathrm{i}}>\nu_{\mathrm{L}}\left(\delta \frac{\Delta \sigma}{\sigma_{0}}-2\right)
$$

instead of equations (31) and (32) of [1]. Also the corresponding growth rate will be $1.12 \times 10^{4} \mathrm{~s}^{-1}$ (for the same plasma parameters as in [1]) whereas it was $1.17 \times$ $10^{4} \mathrm{~s}^{-1}$ in [1]. Hence all these considerations show that there is no qualitative change, but a small quantitative change (the order of magnitude is the same) in the result of $[1]$.

Finally, the source-sink models described above are all fluid models (which are unable to take into account velocity distribution effects, etc.) based on the particular typical laboratory conditions. Hence, it is true that a kinetic model $[8,9]$ would be more appropriate to study the ionization-recombination effectsin dusty plasma. 


\section{Acknowledgements}

The author would like to thank Professor J. Goree and Professor N. D'Angelo of the Department of Physics and Astronomy, University of Iowa, for their valuable comments and constructive suggestions regarding the physics and also experimental conditions of 'formation of voids' and 'ionization instability' in a dusty plasma.

\section{References}

[1] Ghosh, S. 2005 J. Plasma Phys. 71, 519.

[2] Johnson, J. C., D’Angelo, N. and Merlino, R. L. 1990 J. Phys. D 23, 682.

[3] D'Angelo, N. 1997 Phys. Plasmas 4, 3422.

[4] D'Angelo, N. 1998 Phys. Plasmas 5, 3155.

[5] Wang, X., Bhattacharjee, A., Gou, S. K. and Goree, J. 2001 Phys. Plasmas 8, 5018.

[6] Samsonov, D. and Goree, J. 1999 Phys. Rev. E 59, 1047.

[7] Prabhuram, G. and Goree, J. 1996 Phys. Plasmas 3, 1212.

[8] Catto, P. J. 1994 Phys. Plasmas 1, 1936.

[9] Helander, P. Krasheninnikov, S. I. and Catto, P. J. 1994 Phys. Plasmas 1, 3174. 